\title{
PROCEDIMIENTOS Y MÉTODOS PARA LA SISTEMATIZACIÓN DE TRABAJO PSICOSOCIAL EN DESASTRES
}

\section{PROCEDURES AND METHODS FOR THE SYSTEMATIZATION OF PSYCHOSOCIAL WORK IN COMMUNITIES AFFECTED BY DISASTERS}

\author{
Ana Lorena Sáenz Segreda* \\ Adriana Vindas González** \\ Luis Andrés Villalobos Fernández ${ }^{* * *}$
}

\begin{abstract}
RESUMEN
En este artículo se presentan recomendaciones y se comparten conclusiones derivadas de un primer momento de sistematización de las experiencias de la Brigada de Atención de Emergencias y Desastres de la Universidad de Costa Rica. Se espera que sirvan de referencia a personas que realizan trabajo psicosocial en comunidades afectadas por emergencias $y$ desastres, así como a quienes quieran llevar a cabo un proceso de sistematización de sus experiencias. Se considera que la sistematización es fundamental en este tipo de proyectos, ya que no solo sirve para visibilizar los logros que se han alcanzado, sino que es la base para llevar a cabo un proceso de autocrítica que permita mejorar el trabajo que se realiza, de cara a los retos futuros.
\end{abstract}

PALABRAS CLAVE: DESASTRES * COMUNIDADES * PSICOLOGÍA * ACCIÓN SOCIAL

\section{ABSTRACT}

This article presents recommendations and conclusions derived of the initially systematization of experiences of the Brigade of Disaster and Emergency at the University of Costa Rica. It is expected to serve as references to people doing psychosocial work in communities affected by emergencies and disasters, and want to carry out a process of systematization of experiences. The reorganization is essential in this type of project as it not only serves to make visible the achievements that have been made, but is the basis for conducting a self-critical process which improves the work done, in order to future challenges.

KEYWORDS: DISASTERS * COMMUNITIES * PSYCHOLOGY * SOCIAL ACTION

\footnotetext{
* $\quad$ Escuela de Psicología de la Universidad de Costa Rica (UCR). lorena.saenz@gmail.com

** Escuela de Psicología de la Universidad de Costa Rica (UCR). adriana.vindas@ucr.ac.cr

*** Egresado de la Escuela de Psicología de la Universidad de Costa Rica (UCR). psycoluis2@yahoo.com
} 


\section{INTRODUCCIÓN}

La Brigada de Atención Psicosocial en Situaciones de Emergencia y Desastres es un proyecto de acción social de la Universidad de Costa Rica que tiene por objetivo llevar a cabo procesos de recuperación psicosocial en comunidades del país afectadas por desastres $y / 0$ situaciones definidas como emergencias.

Cuando se habla de que la intervención en desastres debe ser psicosocial, significa que la ayuda que se da a las personas afectadas no se puede limitar a subsanar las necesidades físicas básicas como abrigo y comida, sino que es necesario reconstruir el tejido social que conformaba la comunidad, pues aun cuando se logren recuperar los objetos materiales, persiste el sentimiento de pérdida por los que murieron, los espacios comunes desaparecidos y las actividades en conjunto que se perdieron (Beristain, 2000).

Además, implica que al trabajar con las personas se debe tomar en cuenta las interpretaciones que cada una de estas hacen de los hecho vividos, además de como estas orientan su comportamiento y su forma de reconstruir su cotidianidad (Beristain, 2000).

Tras 23 años de labor, era indispensable llevar a cabo un proyecto de investigación que recuperara los distintos aportes de la Brigada a lo largo de los años y que a su vez, produjera insumos importantes para el desarrollo de la Psicología de Emergencias y Desastres en Costa Rica.

Para la ejecución de la labor, se decidió apegarse a la metodología de Oscar Jara (1994 y 2012), la cual proporcionó una base sólida sobre la cual trabajar, sin embargo y tal como menciona este autor, los diversos tipos de experiencias en trabajo comunal que se desean sistematizar poseen matices que las hacen distintas $y$ por tanto, se deben hacer variaciones a la hora de abordarlas.

Tras realizar una extensa revisión en bases de datos, no se encontró ningún artículo que abordara las variables específicas que se presentan al sistematizar proyectos relacionados con la temática de la atención psicosocial en situaciones de emergencias $y$ desastres. Por esta razón, se decidió compartir por medio de este documento, algunas recomendaciones y conclusiones que podrían ayudar a otros $y$ otras profesionales del área de Psicología en Emergencias y Desastres, quienes deseen sistematizar el trabajo que han ido realizando a lo largo de los años.

El presente artículo se referirá a las etapas iniciales del proceso, la totalidad de los resultados se esperan publicar en un libro aun por titular, el cual está dirigido a todos y todas las profesionales que de alguna forma estén interesadas en el tema del desarrollo histórico de la Psicología de Desastres en el país. Se reitera que en el presente texto se encontrarán aspectos metodológicos y teóricos de la investigación en Psicología de situaciones de emergencias $y$ desastres.

\section{¿QUÉ ES SISTEMATIZAR?}

Diversos autores como Jara (1994 y 2012), Fernández (2006) y Chávez-Tafour (2006) definen la sistematización como un procedimiento cualitativo por medio del cual se pretenden recuperar las experiencias llevadas a cabo por una iniciativa de acción social, esto como parte de un proceso de aprendizaje que permita a los involucrados e involucradas en la acción social aprender de los errores del pasado para posteriormente, hacer frente a las dificultades que se presentarán en el futuro: "a través de ella nos interesaba identificar el modo en que se relacionan esos saberes distintos, esos actores diferenciados, la manera como estos interpretan lo ocurrido y los modos de legitimación que instruye cada proyecto" (Torres, 1996: 14).

Las diferentes intervenciones produjeron informes que contienen resultados y reflejan posiciones teóricas y metodológicas con matices distintos a lo largo del proceso. Se requirió de un procedimiento para el acomodamiento de los datos. Se destaca a continuación algunos de los pasos:

1. La revisión del material escrito producido por el proyecto, tanto el publicado en artículos o libros, como el que nunca se pretendió publicar como informes, entrevistas y diarios de campo.

2. Elaboración de matrices para ordenar la información según categorías de análisis que posteriormente, constituirán los elementos claves del análisis. 
3. Entrevistas tanto a los y las profesionales que en diversos momentos históricos han estado vinculados(as) al proyecto, como a las personas beneficiadas por la labor realizada $y$ las diversas autoridades que han tenido alguna influencia en la situación que se está estudiando.

Llevar a cabo el proceso anterior implicó para los investigadores, los siguientes aspectos: mantener una actitud/ganas de desafiar y ser desafiado(a) durante todo el proceso, estructurar y ordenar una propuesta, tener tiempo destinado a la tarea de sistematización, trabajar con $y$ desde la realidad, $y$ ser agentes externos de las experiencias de campo (Jara, 1994).

La ausencia de alguno de los aspectos anteriores definitivamente hubiese significado detener el proceso o intentar llegar a conclusiones más prontas o determinadas por los y las investigadoras, en lugar de permitir que el mismo flujo de los productos de las intervenciones marcaran el punto de llegada y las direcciones a tomar en el proceso de la sistematización.

A continuación se presentan apartados correspondientes a cada una de las etapas del proceso de sistematización realizadas por las $y$ los investigadores. En cada una se explicará en qué consistió, así como, las particularidades que se han presentado en este proyecto de investigación y las cuáles seguramente tendrán que hacer frente los y las profesionales que deseen sistematizar proyectos de trabajo psicosocial en emergencias y desastres.

\section{ETAPA: LA ELABORACIÓN DE UNA PROPUESTA}

Un problema que se suele dar con respecto a los procesos de sistematización de las experiencias de acción social, es que debido a la gran cantidad de trabajo que se realiza con la comunidad, los y las investigadoras carecen del tiempo necesario para realizar una adecuada sistematización de este trabajo. En este caso se reflejó en que la mayoría de los informes encontrados eran breves y respondían a requisitos de las universidades o de los organismos que proveen fondos para este tipo de proyectos.

Los y las investigadoras que dirigen las intervenciones siempre tienen demandas que atender, por ejemplo, la Brigada amplifica su accionar y pasa a lo largo del tiempo de la atención de situaciones de emergencia derivadas de desastres, a la atención de las mismas, más situaciones de emergencia de génesis diversa. Lo anterior significa una demanda permanente de atenciones y por ende, una imposibilidad de tiempo efectivo para los procesos de sistematización de las experiencias por medios distintos a los informes mencionados.

En el trabajo de campo con personas afectadas por desastres $y / 0$ situaciones de emergencia, se presenta una gran cantidad de situaciones que continuamente fuerzan a que se realicen modificaciones a la propuesta original, lo cual es válido desde varios enfoques metodológicos cualitativos (Hernández, Fernández y Baptista, 2006). Sin embargo, en el proceso post intervención, en la sistematización es necesario llevar a cabo una propuesta muy estructurada, la cual permita precisamente que la enorme cantidad de material generado en el trabajo de campo, pueda mostrar claramente sus virtudes $y$ defectos.

La Brigada de Atención Psicosocial en Emergencias y Desastres de la Escuela de Psicología de la Universidad de Costa Rica, siempre ha señalado la necesidad de espacios de sistematización, pero no fue posible hasta ahora contar con profesionales que de forma paralela logren ir registrando la información (debido a la falta de presupuesto y planificación dentro del proyecto mismo). Al revisar las producciones sobre el tema, se encontró que esta problemática no es exclusiva de este proyecto, sino que es la regla general en la mayoría de los proyectos de acción social no solo en nuestro país, sino en Centroamérica en general.

De forma que además de que los y las investigadoras conozcan los requerimientos y tengan la disposición para hacerlo, la acumulación de información e informes durante años, propone al investigador(a) una tarea que realmente constituye un desafío: la forma en cómo se ordenará y destacarán las acciones desarrolladas por las brigadas para constituirlas en insumo de referencia en futuros procesos de sistematización. 
Durante esta etapa, las y los investigadores encargados del proceso de sistematización, deben mantenerse conscientes acerca de la necesidad de apropiarse y familiarizarse con el material, a fin de definir las categorías de trabajo que guiarán la propuesta de sistematización. En el momento en que el equipo de investigadores logra esta armonía entre propuesta, materiales y líneas de desarrollo, se puede afirmar que se establece un punto de partida $y$ que el mismo es coherente con el objetivo principal de los procesos de sistematización de las acciones sociales: dar a conocer tanto los aspectos positivos como los negativos de las intervenciones y construir conocimientos a partir de la reflexión crítica de la recuperación de procesos vividos: "debemos evitar presentar solo ideas positivas, intentando ser imparciales y estando abiertos a la crítica constructiva, un documento que solo presente ideas 0 resultados positivos tendrá muy poca credibilidad" (Chavez-Tafour, 2006: 24).

Para la elaboración de la propuesta es fundamental la consideración del contexto histórico-institucional. En todo proceso de sistematización de experiencias de abordaje psicosocial, se requiere tomar en cuenta el contexto histórico y económico-social en el que se desarrollaron las o la intervención; es decir hay que analizar los procesos históricos, como una complejidad en la que intervienen diferentes actores, que se realizan en un contexto económico-social determinado y en un momento institucional del cual se forma parte (Jara, 2012).

Para explorar este contexto, se centra principalmente en los aspectos jurídicos, pues históricamente, el Proyecto de Atención Psicosocial en Emergencias y Desastres ha sido una instancia dependiente de diversas instituciones estatales que definen los procedimientos a seguir en caso de que ocurra un desastre en el país.

En el año 1999, al amparo de la ley 7914, la Comisión Nacional de Emergencias cambia su nombre a Comisión Nacional de Prevención del Riesgo y Atención de Emergencias, estableciendo la necesidad de que las universidades públicas incorporen en su quehacer universitario contenidos de gestión del riesgo $y$ atención de emergencias. Esto como parte de un cambio paradigmático que sustituye al modelo de atención del ciclo de los desastres con énfasis más emergencista y fisicalista, por uno que incorpora una nueva visión en la cual los desastres pasan a ser concebidos como fenómenos sociales, los cuales están íntimamente ligados a los procesos de desarrollo. Este cambio paradigmático también se puede observar en las intervenciones llevadas a cabo por el Programa de Atención Psicosocial en Emergencias y Desastres; sin embargo, este será insumo de un artículo posterior derivado de este mismo proceso investigativo.

En el 2006, se define la política nacional $y$ se crea el Sistema Nacional de Gestión del Riesgo, en el cual la gestión del riesgo pasa a ser una política pública transversal a todas las políticas de desarrollo y se establece la obligación de todas las instituciones de incluir la temática en su propia planificación y de asignar presupuestos a las actividades relacionadas con el tema del riesgo en desastre.

Al lado de estas acciones $y$ decretos nacionales, acaecen una serie de acciones que permiten fortalecer la presencia y permanencia del proyecto en la UCR. Por lo general, esto sucede con muchas de las iniciativas de impacto popular, pero no siempre se logran destacar estas "coincidencias histórico contextuales".

Como forma de atender las necesidades de la comunidad nacional, la Universidad de Costa Rica empezó a capacitar a finales de los 80 y principios de los 90 del siglo pasado, a docentes de diversas escuelas como Geografía, Trabajo Social, Medicina y Psicología para la atención de situaciones de emergencias.

Posterior a esta capacitación, el área de Psicología Comunitaria se vio impactada por la temática de intervención en crisis y paralelamente desde la Vicerrectoría de Acción Social (vas) de la Universidad de Costa Rica, por intermedio de la profesora Ana Teresa Álvarez, se impulsa el interés por el proyecto de una brigada y así queda inscrito un proyecto de interés universitario como un proyecto de extensión desde la vas. Todas estas influencias cruzadas resultan en un proyecto que empieza en el año 1988, en la Universidad de Costa Rica: se inicia 
el proceso de acción social que hasta nuestros días realiza la Brigada de Atención Psicosocial de Emergencias y Desastres, la cual ha pasado por varios cambios paradigmáticos desde el emergencista/asistencialista, pasando por la gestión del riesgo y en la actualidad, siguen un enfoque de gestión del riesgo centrado en los derechos humanos.

Cada intervención realizada por la Brigada destaca una realidad socio-histórica determinada, pero para efectos de las acciones de la Brigada de Atención de Emergencias y Desastres, se destacarán las acciones nacionales e internacionales que delimitaron las acciones a lo largo del proceso.

\section{ETAPA: ESTABLECER EL PUNTO DE PARTIDA}

Anteriormente, se planteó que el punto de partida se relaciona con la armonía entre propuesta, materiales y desarrollo de la sistematización, la cual constituye el pilar desde el cual se analizarán los insumos y se enmarcarán los hallazgos y las discusiones reflexivas.

Para que los y las investigadoras pueden desarrollar esta fase, la teoría señala que los mismos requieren cumplir con dos requisitos: haber participado de alguna forma en la experiencia que se va a sistematizar y poseer un registro adecuado de esas experiencias. En cuanto a la participación en la experiencia, la investigadora principal de este proyecto ha dirigido todas las intervenciones llevadas a cabo por el Proyecto de Atención Psicosocial a lo largo de su historia, lo cual da un enorme aporte a la investigación; sin embargo, como se mencionó anteriormente, debido a que la sistematización tiene como uno de sus objetivos principales develar los problemas encontrados en las diversas intervenciones, se requiere de una cierta mirada que sea lo suficientemente externa a las intervenciones de acción social, pero lo suficientemente integrado al equipo de trabajo como para conocer sus dinámicas de trabajo y las particularidades de las comunidades intervenidas.

Debido a lo anterior, se requiere además del investigador principal, un grupo de colaboradores que se dedique a apoyar las siguientes funciones (Jara, 1994): $\diamond \quad$ Diseño y conducción metodológica del proceso

$\diamond \quad$ Búsqueda de información

$\diamond \quad$ Ordenamiento de información

$\diamond \quad$ Insumos de reflexión

De acuerdo a las funciones anteriores, así se integra un equipo de trabajo con personas involucradas en algún grado con el proyecto, pero que no están vinculados en el día a día, intentando con esto, lograr una "distancia objetiva" pero a la vez una relación desde el afecto y el conocimiento con la propuesta de sistematización y sus contenidos.

Todos los integrantes del equipo de trabajo deben estar motivados, familiarizados e identificados con las acciones desarrolladas por los proyectos y con las consecuencias sociales los mismos han derivado.

Relacionado con el registro de las experiencias en este proceso de sistematización, se cuenta con un registro de todas las que se han realizado en los últimos 23 años por parte del Proyecto de Atención Psicosocial en Emergencias y Desastres, de los cuales la enorme mayoría no se han publicado en ningún medio académico.

Estos insumos tampoco tienen un orden determinado, pero se sabe que al ser informes, programas $y / 0$ trabajos universitarios, responden a los ejes del quehacer universitario y que se derivan directamente de las acciones formativas y de acción social desarrolladas por la Brigada durante estos años.

El ordenamiento $y$ el establecimiento de la lógica de trabajo, requiere de reuniones de planificación, de contacto con los insumos de las experiencias $y$ de iniciar una primera fase del proceso de manera crítica y reflexiva.

En esta primera etapa del proceso de sistematización, se definirán las líneas que marcarán los resultados de la misma. A continuación, se destacan acciones que se constituyeron como fundamentales para el logro de la tarea.

Para la construcción del punto de partida, es necesario que los y las investigadoras consideren algunas acciones destacadas por expertos en la materia (Jara, 1994). 
Otro aspecto importante es la definición del objetivo de la sistematización, el cual se logra por medio de las preguntas iniciales, que permiten definir de manera más concreta los resultados que se persiguen: "aquí se trata de definir, de la manera más clara y concreta posible, el sentido, la utilidad, el producto o el resultado que esperamos obtener de la sistematización" (Jara, 1994: 103).

En un proceso de investigación como el presente, es fundamental resaltar la importancia del objetivo general: la sistematización de las experiencias del Proyecto de Atención Psicosocial en Emergencias y Desastres, entre 19902012, enfatizando en los ejes de investigación, docencia y acción social.

Existen documentos que refieren a múltiples intervenciones realizadas a lo largo del tiempo, las cuales se organizaron de manera que puedan asociarse con los ejes del quehacer universitario y permitan así una mayor visibilización del impacto, los alcances y los desafíos del proyecto.

Asimismo, esta organización y presentación del material permitirá identificar las correlaciones entre las acciones desarrolladas por la brigada y las políticas nacionales e internacionales intervinientes en el tema.

$\mathrm{Al}$ existir registros de un periodo extenso, lo fundamental consistió en relacionar tales registros con las áreas o ejes a los que respondían. De esta manera se logró esclarecer que en efecto guardan una correlación con los ejes del quehacer universitario, por lo que resultó conveniente retomar los mismos como ejes generales.

El segundo paso consistió en definir qué se integrará dentro de cada uno de los ejes, nuevamente en etapas iniciales esto remite a la revisión de lo que se tiene, de lo que falta y de la manera de acceder y ordenar esta información. En nuestro caso, al lado de la definición de los ejes resultó necesaria la construcción de las matrices para el ordenamiento de la información, las mismas se derivan de los ejes establecidos, pero registran las acciones por años y elementos-categorías de análisis.

En cuanto a la definición del objeto a sistematizar: "se trata de escoger la o las experiencias concretas que se van a sistematizar, claramente delimitadas en lugar y tiempo" (Jara, 1994: 104).

En el caso de proyectos de acción social como este, en el cual nunca se había realizado una sistematización a profundidad, es necesario en este primer momento, incluir la totalidad de las intervenciones realizadas por el programa a lo largo de su historia.

Se ha definido que en este caso el objeto está constituido por la totalidad de las partes, por lo que los resultados iniciales versarán sobre esta totalidad $y$ no enfatiza en ninguna de las partes que lo conforman. La decisión deja "la escogencia de la o las experiencias" que plantea Jara para la segunda parte de este proceso de sistematización: el destacar algunas de las experiencias de abordaje que por sus particularidades, aportará elementos relevantes para posteriores intervenciones en el área.

Esta decisión de dividir una primera sistematización que considera la totalidad de intervenciones $y$ una segunda, la cual enfatiza $y$ profundiza en alguna de las experiencias, se deriva de la necesidad de crear material socioeducativo para la atención de emergencias y desastres en la comunidad costarricense. Estas producciones pueden ser utilizadas por distintas poblaciones $y$ pueden producirse a partir de las acciones e informes existentes de la Brigada de Atención de Emergencias y Desastres de la Escuela de Psicología de la Universidad de Costa Rica.

Finalmente, en lo que se refiere a la precisión del eje a sistematizar, Jara define que "un eje de sistematización es como una columna vertebral que nos comunica con toda la experiencia, pero desde una óptica específica" (1994: 106).

La razón por la que se va a trabajar desde los ejes de investigación, docencia y acción social, es que desde el estatuto orgánico de la Universidad de Costa Rica, se plantea que precisamente estos son sus tres ejes de acción, haciendo que todos sus programas deban tener un impacto en esas áreas, razón por la cual se realza la labor realizada por el Proyecto de Atención Psicosocial en Emergencias y Desastres en esas áreas. Como se señaló anteriormente, los informes con los que se cuenta, responden a estos ejes de trabajo. 
III ETAPA: ELABORACIÓN DEL MARCO REFERENCIAL

El análisis del objeto de sistematización es un paso adjunto a los propuestos por Jara. Se propone que esta fase se debe dar inmediatamente después de que se haya precisado el eje a sistematizar y se centra en la búsqueda de materiales teóricos orientados específicamente a la sistematización de experiencias.

Cuando se realiza la experiencia el marco teórico está dado por el área de incidencia del programa o proyecto ejecutado, pero cuando la experiencia se convierte en objeto de análisis aparecen nuevas demandas teóricas no necesariamente trabajadas durante la experiencia (Ayllón, 2004: 48).

En nuestro caso se toman como base principal los planteamientos propuestos por Alforja para la sistematización de experiencias, los cuales proponen que la sistematización sea un proceso por medio del cual se rescatan experiencias prácticas concretas, así como factores objetivos y subjetivos, para poder así aprender cuáles son las fortalezas y las deficiencias, logrando mejorar el trabajo que se realiza en las comunidades (Jara, 1994).

La elaboración de este marco teórico no concluye en la etapa inicial, el equipo de investigadores debe estar anuente durante todo el proceso a integrar o replantear nuevas referencias que apoyen $y$ validen los hallazgos del proceso de recuperación de las experiencias: "no se deducirán explicaciones desde teorías sino que se elaboran teorías a partir de lo vivido, es el cuestionamiento $y$ la desconstrucción de visiones e interpretaciones que teníamos al vivir la experiencia" (Bickel, 2006: 22).

El programa aparece como respuesta a necesidades contextuales y marcos jurídicos relacionados directamente con el tema de derechos humanos. Además, el proceso y la experiencia de las inserciones realizadas marcan una tendencia a una propuesta teórica coincidente con postulados como el holismo y el materialismo histórico dialéctico.
Por ejemplo, en una visión holista, la historia es entendida como la interconexión entre el pasado, el presente y futuro. El presente es producto del pasado y el futuro es producto del presente. Para construir un futuro diferente $y$ mejor es necesario actuar, así como basarse en lecciones del pasado, para plantear escenarios futuros alternativos (De Souza, 2001).

Podría afirmarse también que los procesos vivenciados en las acciones de la Brigada por los y las brigadistas, se comprenden desde la idea que originalmente Vigotsky planteó para el pensamiento infantil acerca de que "el desarrollo de los comportamientos complejos está caracterizado por complicadas, cualitativas transformaciones de una forma de comportamiento a otra (o como Hegel hubiese dicho, una transformación de cantidad a cualidad)" (1978: 19).

Los y las brigadistas desarrollan habilidades y aptitudes "en las intervenciones" que permiten una atención de calidad y calidez a las comunidades, así como, una formación profesional y humana cualitativamente distinta a la que se puede desarrollar en los espacios físicos académicos: "un enfoque holista es un proceso global, evolutivo, integrador, concatenado, organizado y sucesivo que concibe la formación de los educandos en términos de integración e interrelación como un sistema vivo, dinámico, como una comunidad de aprendizaje" (Díaz et ál., 2005: s.p.).

Otro de los referentes teóricos que resulta una pieza fundamental en el trabajo realizado por la Brigada de Atención Psicosocial en Emergencias y Desastres de la Universidad de Costa Rica, ha sido el enfoque de derechos humanos.

El esfuerzo orientado a articular una nueva ética con políticas públicas a nivel social y judicial $y$ privadas, es lo que se ha denominado como el enfoque de derechos humanos. Este procura construir un orden centrado en la creación de relaciones sociales basadas en el reconocimiento $y$ respeto mutuo y en la transparencia, de modo que la satisfacción de las necesidades materiales $y$ subjetivas de todas las personas y colectividades, sin 
excepción alguna, constituya una obligación jurídica y social (Güendel, 1999: 3).

En la intervención en comunidades afectadas por desastres, el enfoque de derechos humanos implica que se reconozcan las problemáticas $y$ necesidades diferentes de los distintos grupos etarios, étnicos y de género, sin prejuzgar que unos sean más importantes que otros (Mora, 2005).

\section{ETAPA: RECUPERACIÓN DEL PROCESO VIVIDO}

En esta etapa se debe realizar una reconstrucción de la historia: "se trata de aquí de tener una visión global de los principales acontecimientos que sucedieron en el lapso de la experiencia" (Jara, 1994: 111).

Este proceso requiere no solo de ordenar los acontecimientos en el tiempo, sino de reconstruir a profundidad esos hechos, razón por la cual se recurrirá (en los casos que proceda) a entrevistar a quienes desempeñaban una función de líderes comunales en la época en que se realizaron las intervenciones, así como a quienes cumplieron funciones de asistentes en el proyecto.

Es fundamental recuperar no solo el acontecimiento desencadenante de la situación que define el abordaje, sino los elementos histórico-contextuales que le rodean.
No es lo mismo un abordaje en los años 90 que en la primera década del 2000, no solo por el contexto sino porque el proceso mismo de abordaje evoluciona desde lo teórico conceptual, así como, los actores sociales involucrados desarrollan nuevas necesidades y/o particularidades.

En este sentido, como ventaja, el periodo a sistematizar es tan amplio, que permite visualizar estos cambios conceptuales en el tiempo, además profundiza en situaciones o acontecimientos determinados, evidenciando a su vez las áreas en las que la Brigada ha logrado impactar de manera más directa y las que aún constituyen un desafío tanto en abordaje como en construcción de insumos teóricos.

Además la recuperación del proceso vivido implica un ordenamiento y clasificación de la información. Específicamente, en la presente investigación significó más de 20 años de trabajo del Programa de Atención Psicosocial en Emergencias y Desastres, lo que se traduce en docenas de documentos (la gran mayoría sin publicar), los cuales suman miles de páginas. Por lo cual, proponerse a analizar esa información sin hacer antes una clasificación y ordenamiento de la misma, es una tarea imposible. Lo primero que se hizo fue clasificar la información conforme a los tres grandes ejes que conforman el accionar de la Universidad de Costa Rica (cuadro 1).

\section{CUADRO 1 \\ EJES DE ACCIÓN \\ UNIVERSIDAD DE COSTA RICA}

\begin{tabular}{lll}
\hline Docencia & Acción Social & Investigación \\
\hline$\diamond$ Programas de los cursos & $\diamond \quad$ Informes de las intervenciones $\diamond$ Proyectos finales de graduación \\
$\diamond$ Listado de la bibliografía utilizada & llevadas a cabo en las comunidades cuya temática fuera la intervención \\
en los cursos & $y$ tcu & psicosocial en emergencias y desastres \\
& $\diamond \quad$ Informes y evaluaciones de los $\diamond \quad$ Ponencias sobre el trabajo de \\
& talleres y otras actividades de carácter campo, presentadas en congresos \\
& psicoeducativo dirigidas al público en \\
& general & \\
\hline
\end{tabular}

Fuente: Elaboración propia. 
Además, se tomaron en cuenta documentos externos, tales como:

$\diamond \quad$ Las diversas legislaciones existentes en el país desde 1990, con respecto a la temática de la intervención en desastres, así como, otras directrices provenientes de instituciones gubernamentales respecto al tema de desastres.

$\diamond \quad$ Manuales $y$ procedimientos de abordajes psicosociales definidos por la OMS y OPS.

$\diamond \quad$ Directrices oficiales provenientes de la Universidad de Costa Rica que afecten la labor del Proyecto de Atención Psicosocial en Emergencias y Desastres.

Luego, se procedió a construir la matriz de datos, la cual se compone de nueve categorías, con sus respectivas sub categorías. Estas se eligieron porque abordan aspectos que se consideran indispensables a la hora de sistematizar la labor de un proyecto de acción social que trabaje con personas afectadas por emergencias $y$ desastres.

\section{1) Tipos de abordajes}

En los últimos 20 años, se ha modificado en gran medida la concepción que se tenía de los desastres, pasando de un enfoque en que solo se pretendía reparar el daño, a uno que busca además fortalecer a la comunidad en su respuesta ante futuras amenazas y que además, toma en cuenta las particularidades de cada segmento de la población, sobre todo los más vulnerables como mujeres, niños $y$ niñas, $y$ personas adultas mayores.

2) Legislación sobre desastres existentes en el país

La legislación existente sobre desastres en cada país, enmarca las acciones y los alcances del trabajo realizado por las brigadas de atención psicosocial.

3) Habilidades desarrolladas por los y las brigadistas

El desarrollo de los nuevos abordajes ha propiciado que los y las brigadistas deban desarrollar cada vez mayores habilidades para trabajar con la población afectada.

4) Habilidades desarrolladas por las y los supervisores del proyecto

A lo largo de la historia ha sido necesaria la reflexión y reconstrucción de los roles asumidos por las y los supervisores de las Brigadas de Atención Psicosocial en Emergencias y Desastres.

\section{5) Profesionalización del proyecto}

Con el paso del tiempo las autoridades se han dado cuenta de que estas iniciativas necesitan recursos para su profesionalización, ya que durante mucho tiempo el trabajo ha sido llevado a cabo casi por completo por voluntarios y voluntarias.

\section{6) Reacción de las comunidades}

En los últimos años, se ha planteado la necesidad de registrar los procesos de aprendizaje de las propias comunidades, propio de los enfoques de gestión del riesgo que busca que las comunidades se organicen y así generar acciones para mitigar el riesgo.

$$
\text { Trabajo interdisciplinario- }
$$
interinstitucional

Desde los nuevos enfoques se propone que las diversas disciplinas e instituciones deben trabajar en conjunto para integrar sus habilidades, capacidades y recursos para la obtención de resultados óptimos.

\section{8) Lógicas conceptuales y metodológicas}

Como se citó anteriormente, los cambios epistemológicos en la concepción de los desastres y emergencias ha derivado en lógicas conceptuales y metodológicas diversas, las cuales son necesario rescatar para el análisis del proceso de las Brigadas.

\section{9) Factores externos}

El éxito en las intervenciones de las Brigadas se puede ver afectado por factores externos, 
tales como: la falta de apoyo institucional o el surgimiento de nuevas situaciones de emergencia en la misma zona.

\section{CONCLUSIONES}

1) $\mathrm{Al}$ sistematizar experiencias relacionadas con desastres es necesario considerar que las acciones que llevan a cabo las brigadas y voluntarios respondan a la legislación nacional de cada país y que en algunos casos, son las fuerzas armadas las que se encargan de activar y dirigir las acciones correspondientes a las situaciones de desastres y emergencias. Esto implica que existe un encuadre predeterminado por ley que enmarca las acciones $y$ las posibilidades que se pueden llevar a cabo en cada país.

2) En cuanto a desarrollos teóricos: a partir del material clasificado $y$ analizado en un primer momento por medio de su organización en las matrices, se definió en primera instancia, la necesidad de generar producciones teóricas que rescaten estas experiencias; de manera que se espera desarrollar al menos tres propuestas socioeducativas, tres artículos académicos y un texto completo que recopile los principales aspectos del trabajo de la Brigada en cuanto a su historia, sus abordajes, las habilidades desarrolladas por supervisores y estudiantes, el trabajo interinstitucional en el país y el impacto en las comunidades abordadas.

Las propuestas socioeducativas considerarán las temáticas derivadas de informes de proyectos finales de graduación y/o trabajos de módulos; entre los cuales se pueden citar: el proceso de duelo tras un desastre, la atención psicológica en niñez y adolescencia en situaciones de desastre, $y$ la percepción $y$ los factores de riesgo.

3) Acerca del registro de las experiencias, es necesario documentar apropiadamente cada una de las intervenciones que se lleve a cabo; si bien es cierto, tal como se destacó al inicio, lo ideal sería ir sistematizando estas experiencias de manera inmediata, no obstante, cuando las circunstancias no lo permitan, los $y$ las investigadoras sociales deberán guardar registros, que como en este caso, a la postre constituirán material básico para producción de conocimiento de interés social.

Cabe destacar la necesidad de que los documentos escritos que se elaboren, sean almacenados con fechas, autores $y$ en buenas condiciones ambientales; así como procurar poseer un respaldo virtual. Además, la correspondencia entre las acciones llevadas a cabo y los elementos históricos contextuales destacarán la consistencia y relevancia, tanto de la historia del proyecto como de la pertinencia del resultado de la sistematización.

4) Se destacan como limitaciones, las siguientes:

$\diamond \quad$ El hecho de no existir una política de publicación constante en el área de educación popular y/o abordajes comunitarios en cuanto a la atención de emergencias $y$ desastres, existen periodos de varios años en los cuales el personal del área de Psicología de Desastres no publicó ningún artículo en revistas indexadas.

$\diamond \quad$ Debido al limitado y excesivamente estructurado formato de los informes que las universidades $y$ organizaciones internacionales exigen a quienes realizan intervenciones comunales, se pierden muchas de las enseñanzas que se obtuvieron en la intervención por medio de la interacción con los y las habitantes de la comunidad. Por lo que registros más cualitativos de las experiencias deben ser recopilados por medio de entrevistas posteriores a la intervención, perdiéndose valiosa información que se genera en este momento de intervención desde la investigación-acción. 


\section{RECOMENDACIONES}

1) Es necesario tomar en cuenta que las clasificaciones que se realizan son un tanto artificiosas $y$ tienen como objetivo facilitar el manejo del material; por ejemplo, una intervención de acción social puede también tener aspectos de investigación y docencia.

2) Es necesario dejar constancia escrita no solo de los aspectos más formales de la intervención, sino también de las subjetividades de la población. Las vivencias $y$ las experiencias cualitativas, quedan plasmadas en los informes de los estudiantes, pero se pierden en los informes institucionales orientados a metas numéricas, más que al rescate y análisis de los procesos. Estas experiencias constituyen valiosos insumos para la generación de conocimiento desde las intervenciones de investigación acción y gestan la posibilidad de análisis y consideración de elementos emocionales claramente presentes en intervenciones con estas génesis.

3) Debido a lo dicho anteriormente, las entrevistas a las personas que intervinieron en la comunidad, como a quienes recibieron la atención se vuelven un aspecto clave del proceso. Es recomendable que los registros queden por escrito $y / o$ digital en la base de datos de acciones del proyecto a sistematizar.

4) Finalmente, el enunciar las "coincidencias históricas" permite encontrar convergencias entre demandas externas e intereses, así como, respuestas institucionales que validan la pertinencia de las intervenciones $y$ destacan la importancia de la continuidad $y$ permanencia de proyectos sociales, los cuales terminan impactando diversas áreas con el objetivo final de atender $y$ fortalecer el desarrollo de los seres humanos.

5) Se recomienda a las y los investigadores que deseen sistematizar procesos de abordaje psicosociales de atención de emergencias $y$ desastres, utilizar las categorías de la matriz de datos que se emplea, considerando las particularidades de cada proyecto.

\section{REFERENCIAS}

Ayllón, M. "Aportes para el diálogo entre el saber y el hacer en la promoción y el desarrollo social". Seminario sobre Sistematización. Bilbao: España, 2004.

Beristain, C. Apoyo psicosocial en catástrofes colectivas: de la prevención a la reconstrucción. Caracas: Asociación Venezolana de Psicología Social, 2000.

Bickel, A. "La sistematización participativa para descubrir los sentidos y aprender de nuestras experiencias". La Piragüa: Revista Latinoamericana de Educación y Politica 23. 2006: 17-28.

Chávez-Tafour, J. Aprender de la experiencia: una metodología para la sistematización. Lima: Belido Ediciones, 2006.

De Souza, J. “¿Una época de cambios o un cambio de época? Elementos de referencia para interpretar las contradicciones del mundo actual". Publicación Mensual del Instituto Científico de Culturas Indígenas 3 (25). San José, Costa Rica: Fundación ISNAR, 2001.

Díaz, M. y otros. El Holismo. Universidad de Cien Fuegos-Cuba, 2005.

Fernández, B. "La sistematización como estrategia de aprendizaje en el sistema ACERCA: potencialidades y limitaciones desde la práctica". La Piragüa: Revista Latinoamericana de Educación y Política 23. 2006: 88-96.

Güendel, L. La política pública y la ciudadanía desde el enfoque de los Derechos Humanos: la búsqueda de una nueva utopía. San José: UNICEF, 1999.

Hernández, R.; Fernández, C. y Baptista, P. Metodología de la investigación. México DF: Mc Graw Hill, 2006.

Jara, O. Para sistematizar experiencias. San José: Alforja, 1994.

Jara, O. La sistematización de experiencias, práctica y teoría para otros mundos posibles. San José: Alforja, 2012.

Mora, A. "Propuesta para la incorporación del enfoque de Derechos Humanos de la niñez y la adolescencia en la atención de la emergencia y la reducción del riesgo 
ante desastres: un proceso participativo con la Junta Comunal de Upala". [Tesis de Licenciatura en Psicología]. Universidad de Costa Rica, 2005.

Torres, A. "La sistematización como investigación interpretativa crítica: entre la teoría y la práctica". Seminario Internacional sobre Sistematización y Producción de
Conocimiento para la Acción. Santiago de Chile, 1996.

Vigostky, L. Mind in society: the development of higher psychological processes. Harvard University, 1978.

Fecha de ingreso: 07/01/2013 Fecha de aprobación: 08/04/2013 